TERRA. Revista de Desarrollo Local e-ISSN: 2386-9968

Número 7 (2020), 263-284

DOI 10.7203/terra.7.18412

IIDL - Instituto Interuniversitario de Desarrollo Local

\title{
Uso de las redes sociales y la brecha digital en el tejido asociativo inmigrante de España
}

\author{
Jenny Carolina Tovar Parra \\ Dra. en Cooperación y Desarrollo por la Universidad de Valencia (Valencia, España) \\ carolinatovar81@hotmail.com

\section{Javier Ernesto Torralba Vásquez} \\ Máster Internacional en Migración por la Universidad de Valencia (Valencia, España) \\ javiertorralba1976@hotmail.com
}

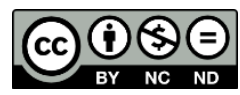

Esta obra se distribuye con la licencia Creative Commons Reconocimiento-NoComercial-SinObraDerivada 4.0 Internacional 


\title{
SECCIÓN NOTAS Y AVANCES DE INVESTIGACIÓN
}

\author{
Uso de las redes sociales y la brecha digital en el tejido asociativo inmigrante de España
}

Resumen: Son innumerables las investigaciones que se han realizado en el mundo sobre los efectos y beneficios de las nuevas tecnologías versus población y la ciudad digital en sus análisis y conclusiones consideran que existe una nueva problemática en el mundo a la que se le denominó "brecha digital”. Sin embargo, no se han realizado investigaciones acerca del efecto que tienen las nuevas tecnologías de información y comunicación TICS sobre el tejido asociativo inmigrante de Argelia, Bulgaria, Colombia, Ecuador, Mali, Marruecos, Rumania y Senegal en España. El objetivo principal de esta investigación es determinar la incidencia de las nuevas tecnologías de la información y la comunicación en el tejido asociativo inmigrante en España, así como conocer el uso de éstas en el quehacer cotidiano de las asociaciones y sus implicaciones para las mismas, partiendo de una metodología no experimental, comparativa, descriptiva y de etnografía virtual. En sus conclusiones queda claro que la brecha digital afecta las asociaciones a partir de la diferenciación entre las que tienen acceso a internet, los conocimientos para el acceso y cuentan con contenidos de calidad, de las asociaciones que no.

Palabras clave: Tecnologías de la información, Redes Sociales, Migración.

\section{Use of social networks and the digital divide in the immigrant community in Spain}

\begin{abstract}
Countless researches have been done in the world on the effects and benefits of the new technologies of information and communication ICT versus population and the digital city in its analysis and conclusions consider that there is a new problematic in the world called "digital divide". However, there are not conducted researches about the effects that ICT has had on the immigrant associations network of Algeria, Bulgaria, Colombia, Ecuador, Mali, Morocco, Romania, and Senegal in Spain. The main objective of this article is to analyze the impact of new information and communication technologies on the immigrant network in Spain, as well as knowing the use of them in the daily work and their implications for them based, starting from a non-experimental, comparative, descriptive and virtual ethnography methodology. In its conclusions is clear that the digital divide affects these associations from the differentiation between those with internet access, the skills to access and the quality on the contents, from those without internet access.
\end{abstract}

Key words: Information Technologies, Social Networks, Migration.

Recibido: 01 de noviembre de 2020

Devuelto para revisión: 04 de diciembre de 2020

Aceptado: 23 de diciembre de 2020

\section{Referencia / Citation:}

Tovar, J. C., y Torralba, J. E. (2020). Uso de las redes sociales y la brecha digital en el tejido asociativo inmigrante de España. TERRA. Revista de Desarrollo Local, (7), 263-284. DOI 10.7203/terra.7.18412 


\section{INTRODUCCIÓN}

Las nuevas tecnologías no son necesariamente sinónimos de progreso y sostenibilidad, aunque esa sea su pretensión. La relación no es directamente proporcional, contraria a lo que se espera. Las Tecnologías de la Información y Comunicación (TIC) están afectando el desarrollo de los países y de su población, en especial en aquellos sectores que aún se encuentran incomunicados a causa de las evidentes dificultades en la implantación de infraestructuras necesarias para la adquisición de esas tecnologías, además de los altos costes y sobrecostes impuestos por los distribuidores y revendedores.

Factores como las amplias zonas geográficas donde no llega ningún tipo de conexión, la situación geográfica, falta de formación y educación de la población, ponen en riesgo la incorporación de estas sociedades a la ciudad digital.

La preocupación actual de los países recae en la adquisición y despliegues de infraestructura y en el ancho de banda necesario para conectar el mundo, minimizando el papel que sobre ello tiene la formación, capacitación y educación digital de la población en general.

En la actualidad las nuevas tecnologías condicionan la evolución y la forma de acceder a los contenidos, de modo que su implantación se ha convertido en sinónimo de desarrollo. En Europa el grado de evolución se mide por el grado de implantación y desarrollo de los veinte servicios básicos definidos en el programa e-Europe 2005, y que van desde el egobierno, e-administración, e-sanidad y e-educación (Comisión Europea, 2010).

Ello supone la digitalización de los trámites públicos, en general a través de sedes electrónicas y páginas web gubernamentales. Esta condición relativamente joven en España obliga a las personas en general y a las asociaciones de inmigrantes a hacer uso de tecnologías con el objetivo de "mejorar", sin contar que muchas de esas personas y asociaciones no cuentan con los tres elementos básicos necesarios en la inclusión digital como: el ordenador, acceso a la red y el dominio de esas herramientas.

Esta situación no es exclusiva de un grupo, sino que afecta a toda la población generando procesos de exclusión socio-digital. Al respecto, muchas de las asociaciones de inmigrantes y sus usuarios en la actualidad dependen de aquellas instituciones y personas capacitadas que cuentan con los medios para hacer uso de las tecnologías.

Nuevamente nos encontramos con que la capacidad para hacer global al mundo y competir en el mercado no es una posibilidad real. En la actualidad, este objetivo se encuentra bastante lejano y más cuando gran parte de África, Oriente medio y algunos países en Asia y América Latina aún se hallan medianamente conectados al mundo virtual a las TIC (Tovar, 2013)..

La desconexión se ha convertido en una verdadera preocupación para los grandes organismos, quienes están destinando fuertes cantidades de dinero con el objeto de contribuir al desarrollo social, mundial, en procura del alcance de los Objetivos de Desarrollo Sostenible (2016-2030). La inversión en infraestructura y la innovación constituyen uno de los 17 Objetivos Globales de la nueva Agenda para el Desarrollo Sostenible.

El énfasis se sustenta en el Objetivo 9 "Industria, innovación e infraestructura", subrayando la necesidad de invertir en infraestructura, industria, tecnologías de información y comunicación, como motores fundamentales del crecimiento y el desarrollo económico, responsable de soluciones permanentes a los desafíos económicos 
y ambientales, así como de la generación de nuevos empleos en el ámbito del desarrollo sostenible. Solo de esta forma será posible reducir la brecha digital como garantía de acceso igualitario a la información y al conocimiento.

A partir del fortalecimiento de los medios de ejecución y el compromiso entre la alianza mundial y la cooperación con el sector privado, se debería dar acceso a los beneficios de las nuevas tecnologías, especialmente las de información y comunicación, tal y como lo sugiere el objetivo 17 titulado “Alianzas para los objetivos”. Dentro de esta alianza se debería incluir el fomento del asociacionismo mundial a través de las telecomunicaciones y de la ayuda de todos los entes públicos y privados necesarios para su existencia y buen desarrollo, como lo sugieran también los anteriores Objetivos del Milenio.

Al respecto, el plan de acción de Hyderabad 2014 reconoce que el incremento en la tecnología refleja el mundo en desarrollo, mientras que la Organización Mundial del Comercio, citada por Mochón (2006), señala que "la tercera revolución industrial será fruto y motor de las nuevas oportunidades tecnológicas y del libre comercio a escala global”, enfatizando en que las nuevas tecnologías harán posible anular las brechas de pobreza y de falta de recursos existentes, en especial en el campo de la economía, de las transformaciones políticas y sociales, de la identidad en la cultura y en el poder, modelando nuevas relaciones a escala global. El problema continúa cuando estos beneficios no están al alcance de todos, y cuando aumentan o disminuyen en procura de los intereses de los grandes monopolios.

La investigación analiza y describe la incidencia de las nuevas tecnologías de la información y comunicación sobre el tejido inmigrante en España y el uso de las mismas en el quehacer diario de las asociaciones.

Partiendo de la hipótesis principal de que las asociaciones de inmigrantes hacen un uso parcial de las nuevas tecnologías que no les permite desarrollar todo su potencial, lo que dificulta su integración en la sociedad de la información desde sus tres ámbitos clave: acceso a dispositivos, conocimientos adecuados y conexión a la red.

Al mismo tiempo, la situación de crisis socioeconómica y política actual del país ha repercutido en el tejido asociativo inmigrante en España, al punto de constituirse en uno de los obstáculos para la integración de las TIC.

Creemos que las redes sociales son utilizadas por las asociaciones de un modo limitado para promocionar y divulgar las acciones, actividades e iniciativas que actualmente desarrollan en España. En cambio, la incorporación de las nuevas tecnologías por las asociaciones de inmigrantes es determinante para el futuro de las mismas y su inclusión o exclusión en la sociedad española y las sociedades de origen de los mismos inmigrantes.

Para conseguirlo se utilizó una metodología no experimental, descriptiva, con análisis combinados entre metodología cuantitativa y cualitativa, apoyada en la etnografía virtual, y se procedió a una revisión bibliográfica que lograra ayudar a comprender y sustentar los resultados encontrados.

Partimos de un análisis de cada una de las asociaciones, enfatizando en los datos de contacto considerados principales indicadores de la adquisición de las TIC, como teléfono fijo, móvil, fax, y e-mail. Posteriormente procedimos a la búsqueda de cada una de las asociaciones a través de internet con el objetivo de verificar el uso de estas herramientas, centrándonos en páginas web, blogs y en redes sociales como Facebook, Twitter y en canales de YouTube. 
Aunque esas herramientas hacen parte de las nuevas tecnologías, cada una de ellas lleva impresas unas características, conocimientos y unos fines diferentes que se intentarán describir del modo más exacto posible en sus particularidades.

Esta investigación cobra especial importancia en España como país de destino. A causa de ese acelerado cambio migratorio, España se ha visto enfrentado a un contexto para el cual no estaba preparada, además coincidió con la crisis económica actual. Esta situación se une a los cambios normativos, que los organismos nacionales e internacionales vinculantes con la migración impulsan. Este tipo de iniciativas respaldadas por las investigaciones actuales, intentan mejorar el entorno, las políticas de integración e inmigración y fomentan a su vez estrategias de desarrollo y cooperación entre los países implicados.

De hecho, el Plan Director de Cooperación Española (2013-2016) reconoce que para diseñar una política es necesario tener una idea clara del país, conocer el contexto en el que se va actuar e identificar con claridad cuáles son los valores añadidos que se pueden aportar, así como destaca la importancia que tienen las tecnologías de la información y las comunicaciones en los procesos de toma de decisiones.

Reconociendo, que existe una nueva realidad que nos sitúan ante un marco totalmente distinto al de hace unos años, que involucra la globalización, los fenómenos migratorios, el cambio climático, el contencioso agrícola, las nuevas tecnologías, el auge de los países emergentes, la aparición de nuevos actores de cooperación que necesita nuevas respuestas y para el que se deberá estar preparado.

Simultáneamente, dentro del contexto de la Unión Europea, España hace aproximadamente diez años viene desarrollando diferentes programas con el objeto de profundizar en la implantación de la Sociedad de la Información. Esta transformación está impulsada principalmente por los nuevos medios disponibles para crear y divulgar información mediante tecnologías digitales. Los flujos de información, las comunicaciones y los mecanismos de coordinación se están digitalizando en muchos sectores de la sociedad, proceso que se traduce en la aparición progresiva de nuevas formas de organización social y productiva.

Esta actividad digital, se está convirtiendo en un fenómeno global que se originó especialmente en las sociedades industrializadas más maduras. La adopción de este modelo basado en la tecnología se relaciona íntimamente con el grado de desarrollo de la sociedad. Sin embargo, la tecnología es fruto y consecuencia del desarrollo y también es una herramienta que deberá estar al alcance de todos y que hoy por hoy puede constituirse en un obstáculo para el inmigrante quien deberá coexistir con las exigencias de este nuevo tipo de sociedad, estar informados y actualizados, innovar, pero sobre todo generar propuestas y generar conocimiento, que surge de los millones de datos que circulan en la red.

Este estudio de la inmigración es importante, porque conocer sus colectivos, así como sus necesidades y la relación con el uso y el acceso que estos tienen con las nuevas tecnologías ayudará a tomar decisiones más puntales y acertadas; además, es de recordar que el tema de la migración en España y del acceso a las nuevas tecnologías cobra cada vez mayor importancia en el área política y en el destino de los futuros gobiernos, quienes también comienzan a reconocer el papel de los inmigrantes, sus asociaciones y la inmersión de los mismos en las nuevas tecnologías, así como la existencia de la brecha digital. 
De hecho, este ha sido uno de los temas principales a abordar en los objetivos del desarrollo sostenible (2016-2030) y de la estrategia de crecimiento de la Unión Europea “Europa 2020”.

En la actualidad es limitado el número de estudios que combinen enfoques descriptivos y comparativos que tomen de referente los países que aquí se abordan y que intenten al mismo tiempo conectarlos con las nuevas tecnologías a través de una etnografía virtual enfatizando en ambos países origen y destino, en especial porque el tema de las nuevas tecnologías es novedoso y las investigaciones que se han realizado priman sobre los sujetos en particular y no sobre asociaciones de inmigrantes como puentes de comunicación entre el país de origen y destino.

Considerando que la incorporación de las nuevas tecnologías por las asociaciones de inmigrantes es determinante para el futuro de las mismas y su inclusión o exclusión en la sociedad española y las sociedades de origen de los mismos inmigrantes.

La finalidad se focaliza en conocer el asociacionismo inmigrante en España, e incorporar la brecha digital desde sus tres ámbitos claves: acceso a dispositivos, conocimientos adecuados y conexión a la red.

\section{METODOLOGÍA}

Este articulo forma parte de una investigación doctoral realizada desde septiembre de 2012 a diciembre 2015. Dicha investigación analiza las características del tejido asociativo inmigrante en España partir de ocho países (Argelia, Bulgaria, Colombia, Ecuador, Marruecos, Mali, Rumania y Senegal). El objetivo principal fue determinar la incidencia de las nuevas tecnologías de la información y la comunicación en el tejido asociativo inmigrante en España, así como conocer el uso de éstas en el quehacer cotidiano de las asociaciones y sus implicaciones para las mismas.

Objetivos específicos:

i) Conocer las principales características de las asociaciones de inmigrantes de los países que forman parte del estudio.

ii) Profundizar en el manejo de las nuevas tecnologías de la comunicación y la información por parte de las asociaciones (redes sociales, páginas web, blogs y otras herramientas).

iii) Determinar las implicaciones del empleo de las nuevas tecnologías sobre el trabajo de las propias organizaciones y su proyección social. Discutir los efectos del acceso a las nuevas tecnologías y la brecha digital sobre las asociaciones de inmigrantes de diferentes países.

La hipótesis principal es que las asociaciones de inmigrantes hacen un uso parcial de las nuevas tecnologías, lo que no les permite desarrollar todo su potencial organizativo, dificultando su integración en la sociedad de la información desde sus tres ámbitos clave: acceso a dispositivos, conocimientos adecuados y conexión a la red.

Para conseguirlo se utilizó un diseño no experimental, comparativo, descriptivo de enfoque mixto y la etnografía virtual, con el objetivo de utilizar la fortaleza de ambos tipos durante toda la investigación. La primera etapa de la investigación se inicia con la recolección de datos utilizando todas las fuentes disponibles: registros de entidades 
sociales y registros autonómicos, listados del Registro Nacional de Asociaciones, censos de asociaciones y listados elaborados por los consulados.

Con esos registros se crea la base de datos inicial constituida por 852 registros de asociaciones, luego se depura y delimita la muestra a partir de los siguientes criterios:

Tenía que tratarse de asociaciones fundadas por inmigrantes, pertenecientes a los países de estudio, legalmente constituidas, activas al momento de la aplicación y sin vínculos religiosos, de esta forma la muestra se redujo a 216 asociaciones.

Posteriormente, se diseñó y aplicó un cuestionario con 21 preguntas cerradas y de selección múltiple, cuyo objetivo era conocer el estado actual y las características de las asociaciones de inmigrantes, en España.

En la segunda etapa de la investigación se buscó evaluar la incidencia y el uso de las redes sociales sobre el tejido asociativo inmigrante.

Para lograrlo agregamos un nuevo criterio: las asociaciones tenían que tener al menos una cuenta activa a nombre de la asociación en alguna de estas redes sociales: Facebook, Twitter, Blog, Web, YouTube.

Obteniendo así 116 registros de asociaciones que cumplían con el criterio. Para su evaluación se diseñaron cinco listas de chequeo a ser administradas por la misma investigadora en la revisión de cada una de las páginas así: Lista de chequeo para Facebook: con 28 preguntas; Lista de chequeo de Twitter con 16 preguntas; Lista de chequeo de YouTube con 8 preguntas; Lista de chequeo para páginas Web con 27 preguntas y Lista de chequeo en los Blogs con 22 preguntas.

La validación de todos los instrumentos se hizo a través de un grupo de expertos externos y luego se utilizó un grupo piloto para la aplicación inicial de los instrumentos. La aplicación de los instrumentos fue realizada directamente por la investigadora a través del uso de la web 2.0 y de los diferentes buscadores y páginas sociales.

\section{BRECHA DIGITAL}

El concepto de brecha digital se enraíza en el llamado informe "El eslabón perdido" de Maitland (1984), que se publicó por la Comisión Maitland en 2003. Este informe advierte en sus conclusiones el "enorme desequilibrio del acceso telefónico entre países desarrollados y en desarrollo”, en especial por la carencia que se tenía en infraestructuras de telecomunicaciones. Su tesis yacía en el número de líneas telefónicas fijas en el mundo, puesto que para ese momento era la principal tecnología de comunicación existente, considerando que el burdo y creciente desequilibrio del reparto de las telecomunicaciones por todo el mundo no se podía tolerar, dado que, en aquel momento, "tres cuartas partes de los 600 millones de teléfonos que había en el planeta se concentraban en sólo nueve países industrializados”.

Maitland, consideraba que el ritmo acelerado de la tecnología es excluyente, puesto que "solo los habitantes del mundo industrializado podrán beneficiarse de la llamada ‘sociedad de la información' para finales del siglo XX”, y que la situación en el mundo en desarrollo contrastaba radicalmente con la de los países desarrollados.

En el informe se reitera la idea de que "en la mayoría de los países en desarrollo, el sistema de telecomunicaciones es inadecuado para sostener servicios esenciales. En extensas 
zonas territoriales no existe ningún sistema. Y tal disparidad no es aceptable en nombre de la humanidad ni sobre la base del interés común”.

A estas conclusiones ya habían llegado antecesores como Wiener (1948), Bell (1973) y Drucker en (1974), quienes para ese tiempo ya habían anunciado los efectos negativos que tendría en la población la nueva sociedad del conocimiento y las tecnologías de información y comunicación, sino se comenzaba a trabajar en formación, capacitación e implantación de tecnologías, para hacer frente a ese nuevo cambio.

Aun así, la autoría del término Brecha Digital no tiene una ubicación precisa, aunque se adjudica a (Lloyd, citado por Rodríguez, 2012), como el primero en utilizar este término en los Estados Unidos durante el gobierno de Clinton para sino se superaban serias diferencias entre territorios, razas, etnias, clases y géneros, mediante inversiones públicas en infraestructuras y ayudas a la educación.

A raíz de ello, se comenzó a generar una serie de publicaciones bajo el título "Falling through the Net”, donde se documentaba la escasa habilidad o competencia de un gran sector de la población, especialmente entre aquellas personas con edades avanzadas para manejar las herramientas tecnológicas de computación y cuyo acceso a los servicios de internet era muy escasos, y para describir como este problema afectaba seriamente la sociedad estadounidense y a la sociedad en general, puesto que los efectos se iban a convertirse en un fenómeno global.

En la actualidad, los estudios más recientes sobre el tema no solo evalúan el acceso a internet, sino que se tiene en cuenta la calidad de dicho acceso y la disponibilidad de conexiones de banda ancha que permitan acceder a contenidos multimedia en tiempos y costos adecuados al contexto de los usuarios.

Como el estudio realizado por el investigador holandés Van Dijk (2001), identificó cuatro dimensiones en el acceso a las tecnologías y la red; estas son: la motivación para acceder, el acceso al material, las competencias para el acceso, y el acceso para usos avanzados. Para Van Dijk "la brecha digital está en constante evolución, dado el surgimiento de nuevos usos tecnológicos, que son apropiados más rápidamente por aquellos que tienen el acceso en forma más permanente y de mejor calidad, determinado por dicho ancho de banda”. Las cuatro dimensiones son necesarias a la hora de formar parte de la Ciudad Digital y las TIC.

De hecho, gran parte de los discursos disfrazan los problemas en ventajas proyectivas. Por ejemplo, la "Cumbre Mundial sobre la Sociedad de la Información” (CMSI), (2005) enfatizó en el papel de las nuevas tecnologías como herramientas de comunicación de valores comunes entre grupos, individuos y organizaciones sociales, criticando la percepción instrumental que se tiene sobre las tecnologías, percibidas principalmente como un fin más que como un medio para impulsar un cambio social.

Una visión instrumental que no considera ni las barreras culturales ni lingüísticas, ni las relaciones de dependencia y subordinación técnica, económica y política entre y dentro de países conectados y no conectados en el mundo.

El llamamiento introduce otro concepto que se supone es el fin que persigue las TIC, y no es otro que la inclusión digital, considerada como un asunto colectivo, no individual, en donde los beneficios sociales deben evaluarse con base en resultados medidos bajo la proyección e involucramiento de los beneficiarios, que en últimas son las comunidades, asociaciones, familias y grupos que sacan provecho de las tecnologías, siempre y cuando tengan acceso a éstas. 
Como se menciona anteriormente, la inclusión digital precisa de tres instrumentos básicos: el ordenador, acceso a la red, y el dominio de esas herramientas. Por lo tanto, no basta con tener un ordenador conectado a internet para considerar incluida digitalmente a una persona; se precisa saber qué hacer con esta tecnología y cómo utilizarla, lo que supone capacitación y conocimiento.

En este sentido, la eficacia de las políticas de inclusión digital dependerá de las posibilidades de integración del conocimiento en los propios objetivos de sus beneficiarios, considerando que no existe un solo modelo, sino muchas modelos de posibles sociedades de la información y de la comunicación adaptadas según las diferentes necesidades, objetivos individuales y comunes en cada país.

También se tendrá en cuenta la diferencia socioeconómica entre aquellas comunidades que tienen accesibilidad a Internet y aquellas que no, aunque tales desigualdades también se pueden referir a todas las nuevas tecnologías de la información y la comunicación TIC, como el uso de portátiles, la telefonía móvil de tercera y cuarta generación, la banda ancha y otros dispositivos.

Para efectos de esta investigación, en el tejido asociativo se entenderá por brecha digital las diferencias previas en el acceso a las tecnologías, las diferencias que hay entre asociaciones según su capacidad para utilizar las TIC de forma eficaz y la diferencia de recursos entre aquellas asociaciones que tienen accesibilidad a internet y aquellas que no.

Podemos diferenciar tres clases de brecha digital:

i) Brecha Digital de acceso: dificultad que puede tener una persona para disponer de un ordenador conectado al internet, incluyendo las redes avanzadas como tener servidores.

ii) Brecha Digital de uso: diferencia entre llegar a tener acceso a la tecnología y el de saber usar la tecnología.

iii) Brecha Digital de Calidad de uso: se basa en la limitación o la falta de posibilidad para que las personas tengan acceso a conexiones de calidad como la banda ancha y las herramientas que proporciona para conectarse y utilizar los recursos de la red como por ejemplo las sedes electrónicas, redes sociales, negocios e intercambio de información.

Estas tres clases de brecha digital están constituidas a su vez por ocho subniveles que deberán de ser considerados por su relación directa. Estos son 1) nivel de ingreso; 2) nivel educativo; 3) brecha de género; 4) localización geográfica; 5) edad 6) banda ancha; 7) conectividad; y 8) usos de las TICS.

Las clasificaciones existentes se comportan como un abismo que separa a los individuos logrando generar situaciones de exclusión y estratificación socio digital, ya que no es la típica exclusión social entendida como la falta de participación de segmentos de la población en la vida social, económica y cultural de sus respectivas sociedades debido a la carencia de derechos, recursos y capacidades básicas que hacen posible una participación social, sino de exclusión digital capaz de limitar la participación plena de todos los ciudadanos en igualdad de condiciones, limitando el acceso a las nuevas tecnologías (infraestructuras insuficientes), así como la accesibilidad mediante la formación y la educación. Estos dos tipos de exclusión se comportan de diferentes maneras, pero acentúan su existencia, puesto que la exclusión social hace referencia a la identidad de sujeto, mientras que la exclusión digital se refiere a las carencias del sujeto. Solo en la exclusión digital se puede encontrar que existen sectores incluidos socialmente, 
pero excluidos digitalmente y viceversa, generando una posición de inferioridad capaz de multiplicar los niveles circunstanciales de los que están fuera y los que están dentro; La relación es directamente proporcional a medida que aumentan las TICS se aumenta la brecha digital, por tanto a la hora de valorar la brecha digital se debe de tener en cuenta el poder que ejercen las instituciones públicas y privadas, ya que en sus intentos por conciliar intereses crearon un nuevo fenómeno denominado la ciudad digital.

La ciudad del siglo XX, conlleva el impulso de las sociedades digitales y la implantación de lo que se considera la sociedad de la información, esta práctica viene promovida por la Comisión Europea como iniciativa que busca mejorar el funcionamiento de la administración, de los servicios públicos, los procesos democráticos reforzando las políticas públicas, por medio de la difusión de la integración de cambios organizativos y nuevas habilidades con el claro fin de mejorar los tiempos de respuesta, la satisfacción de sus usuarios y el desarrollo sostenible. En el VI Sexto Congreso de sociología 2008 señala que en España los conceptos que sobre la ciudad digital se manejan se enfocan en la necesidad de disminuir el retraso en materia de nuevas tecnologías de la información y la prisa que se tiene por su desarrollo, considerando que las nuevas tecnologías insertan positivamente un factor de desarrollo administrativo y económico en las ciudades. Según el Ministerio de Industria, Turismo y Comercio, "el programa Ciudades Digitales” está concebido para ayudar a las entidades locales, a la inmigración en general, a sus ciudadanos en particular mediante la aplicación de servicios avanzados de telecomunicaciones y de nuevas tecnologías tanto en el marco social como en el económico y de migración.

Los trámites telemáticos públicos y privados van desde el gobierno electrónico, hasta los portales de empleo y migración, cuyo objetivo principal es garantizar la inclusión digital minimizando costos, masificando resultados, e incrementado la satisfacción del cliente, sin embargo nos encontramos con la otra cara de la realidad y en ella se encuentra los "beneficiados", este grupo de personas bien podrían llamarse "afectados", puesto que en cuestión de minutos se han visto rodeados por las nuevas tecnologías, trámites telemáticos, infraestructuras desconocidas y todas las exigencias socio económicas y de pensamiento que ello conlleva.

Es en ese momento en donde las personas comienzan a experimentar procesos de exclusión y de dependencia de aquellos que cuentan con las herramientas informáticas y el conocimiento para realizar el trámite o consulta de algún tipo de estos servicios, en especial porque en la actualidad no es posible realizarlos por otra vía, como, por ejemplo: las renovaciones del paro, solicitudes de permisos de residencia, la nacionalidad etc.

Por lo general esta dependencia queda en manos de algún familiar o amigo y/o de personal externo que cobra por su servicio, de forma que las personas deberán de pagar por la ayuda que recibe aun cuando se supone que dichos trámites no deberán de generan costes en el usuario.

El gobierno está habilitando zonas wifi, colocando ordenadores e incluso impresoras en centros públicos como LABORA (antiguo SERVEF), INEM, bibliotecas, ayuntamientos, la Seguridad Social, etc. Sin embargo, estas medidas aún son minúsculas para la demanda existente y aunque logran cubrir una buena parte de la problemática digital se debe de tener en cuenta que los medios materiales y tecnológicos por sí solos no sirven, se necesita tener el conocimiento y la capacidad para acceder a dichos tramites.

Ahora bien, en el caso de los migrantes esta situación ha supuesto otra barrera y más cuando existen colectivos que por su situación socio-económica, cultural y del idioma no saben o no pueden acceder a dichos trámites, muchos de estos afectados acuden a las 
asociaciones de emigrantes queriendo subsanar sus carencias, pero se encuentran con un nuevo problema y es que el tejido asociativo emigrante en España ha cambiado e incluso está desapareciendo, entre otras causas por la difícil situación socio-económica y estructural por la que atraviesa el país y a los cambios administrativos e ideológicos actuales, ¿a qué nos referimos? Muchas de las asociaciones de emigrantes vivían y se mantenían de las subvenciones públicas obviando la importancia de gestionar sus propios gastos y de abrir su visión a otro tipo de entornos entre ellos las TICS, las razones son innumerables, pero se destaca la falta de conocimientos, formación y de empoderamiento empresarial, puesto que en general estas organizaciones se les dificulta verse como empresas que necesitan auto gestionarse haciendo uso de las nuevas tecnologías.

\section{ANÁLISIS Y DISCUSIÓN DE RESULTADOS}

El objetivo de esta investigación fue determinar la incidencia de las nuevas tecnologías de la información y la comunicación en el tejido asociativo inmigrante de ocho países (Argelia, Bulgaria, Colombia, Ecuador, Marruecos, Mali, Rumania y Senegal) en España, así como conocer el uso de éstas en el quehacer cotidiano de las asociaciones y sus implicaciones para las mismas.

La crisis socioeconómica, las políticas gubernamentales, los recortes en protección y el desamparo de los inmigrantes irregulares son los directos responsables de que el papel del inmigrante y de sus asociaciones como agentes dinamizadores de procesos pierdan importancia en España, provocando el cierre y la disminución del número de asociaciones de inmigrantes. De hecho, muchas de estas que participaron en esta investigación en la actualidad han cesado su actividad.

Años atrás eran las propias asociaciones de inmigrantes las que se encargaban de gestionar sus colectivos, en la actualidad su labor se está limitando a la derivación de personas a entidades con mayor trayectoria o del gobierno. Aunque el tejido asociativo afirme realizar y prestar todas las actividades y servicios relacionados con integración social, a la luz de los resultados, queda claro que el papel desempeñado por la mayoría de las asociaciones se reduce al acompañamiento, remisión y seguimiento de sus asociados.

La falta de planeación y formación continuada de los líderes de las asociaciones en temas relacionados con administración de negocios, autofinanciación y las nuevas tecnologías; junto con la dependencia de estas organizaciones de los fondos y subvenciones del gobierno, hicieron que las organizaciones limitaran la creación de planes, programas y proyectos propios, en procura de la generación de ingresos que les garantizara continuidad. Además, entre los inmigrantes se creó la imagen de "servicio gratuito". Situación que junto con las anteriores son las responsables de los cierres de las asociaciones y de que los usuarios se nieguen a pagar por los servicios que reciben, y en el caso de que decidan hacerlo, lo harán en el centro que más y mejor cobertura les provea, de ahí la competencia. En cuanto al uso de las Tics por parte de las asociaciones encontramos: 
Figura 1. Asociaciones encuestadas a nivel nacional y con redes sociales

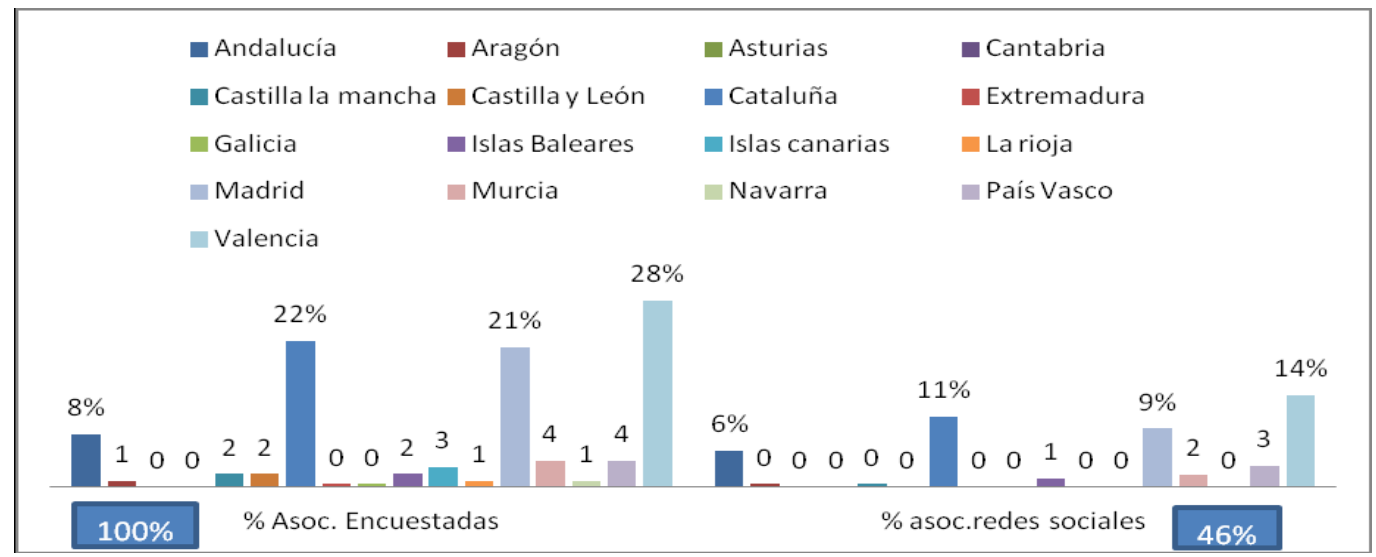

Fuente: de elaboración propia.

Que el uso que hacen las asociaciones de inmigrantes de las tecnologías de información y comunicación (TIC) a nivel general es más bien escaso (Figura 1), pues solo el 46\% del total de las asociaciones cuentan con algún tipo de presencia en la web.

En términos generales, los flujos migratorios están más asentados en las principales ciudades de España, de ahí que su incorporación a la web 2.0 y que el acceso a internet sea mayor, en especial porque el desarrollo en telecomunicaciones y en conexiones de internet también se concentra principalmente en las grandes ciudades.

La falta de recursos económicos ha hecho, que el grueso de las asociaciones de inmigrantes carezca de locales propios o alquilados para el desarrollo de sus actividades. Razón por la cual, muchas asociaciones han tenido que cerrar sus puertas, y otras han trasladado las asociaciones a sus hogares, convirtiéndolos en centros de reunión y de trabajo.

Figura 2. Tipo de personal en las asociaciones

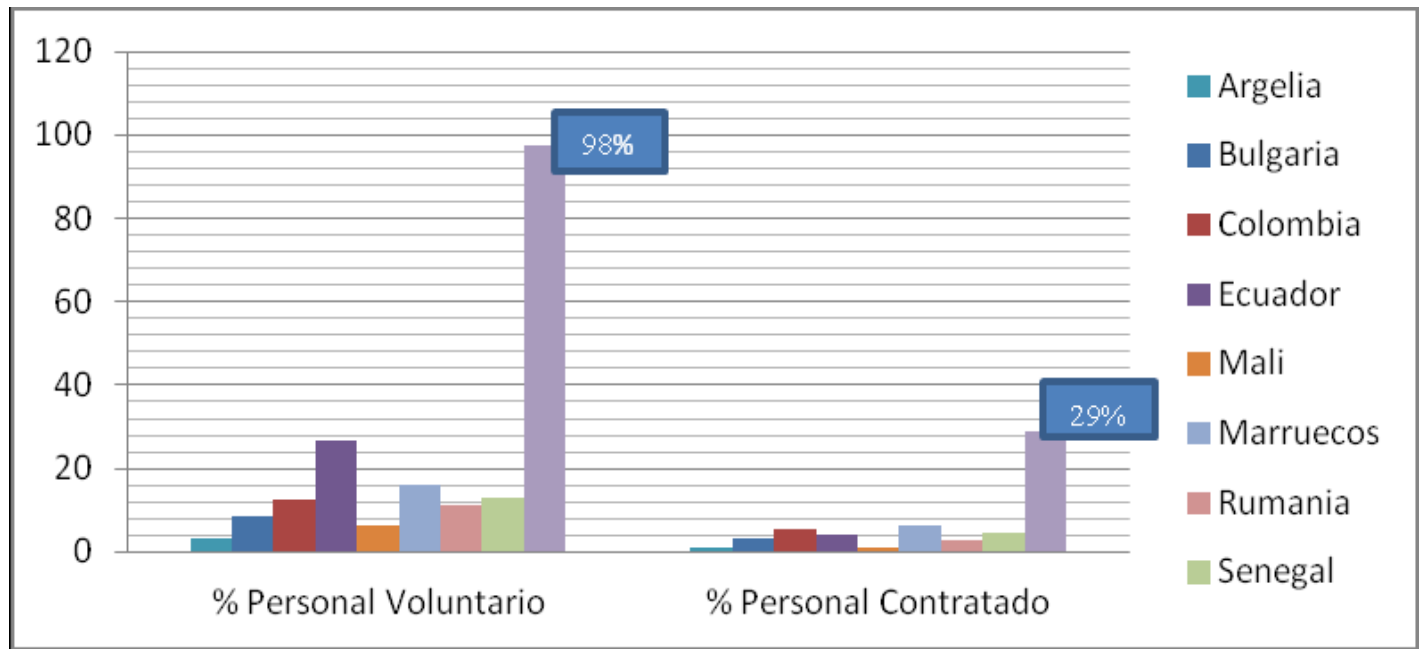

Fuente: elaboración propia. 
En cuanto al personal de las asociaciones se encontró que en su mayoría era de carácter voluntario y solo un pequeño porcentaje tenía personal contratado, que incluye a los fundadores, quienes hacen funciones polivalentes que van desde la administración hasta actuar como profesores y consejeros, muchas veces sin las titulaciones y conocimientos requeridos (Figura 2).

El 98\% de las asociaciones cuenta con personal voluntario para el funcionamiento y desarrollo de sus actividades, y solo el 29\% tiene personal contratado incluyendo a sus fundadores.

Aunque el papel que viene desempeñando el voluntario en estas instituciones es de vital importancia no se puede pensar que a partir de este personal la institución podrá salir adelante, en especial si se considera que el voluntario es más bien un personal inestable.

Debido a esta situación, las asociaciones de inmigrantes que aún existen se encuentran en desventaja profesional con aquellas organizaciones donde tienen personal preparado, capacitado y contratado que da apoyo y que cuentan con sus propios psicólogos, contables, etc. Este es también el motivo por el cual las asociaciones solo se dedican a derivar a sus asociados a otros centros de atención y organizaciones no gubernamentales como Cruz Roja, Caritas y los centros de atención a la inmigración de los ayuntamientos.

Las tecnologías líderes para comunicarse siguen siendo las tecnologías de antaño, las líneas móviles y fijas, que están condicionadas a los medios económicos de la asociación. Se ha primado la estrategia del uso de recursos personales a disposición de la asociación, dado que en términos generales no se tiene infraestructura propia (Tabla 1).

Tabla 1. Tecnologías de información y comunicación dentro del tejido asociativo

\begin{tabular}{|c|c|c|c|c|c|c|c|c|c|c|c|c|c|}
\hline \multirow[b]{2}{*}{ País } & \multirow{2}{*}{$\begin{array}{c}N^{\circ} \\
\text { de } \\
\text { Aso. }\end{array}$} & \multicolumn{6}{|c|}{ Líneas telefónicas } & \multicolumn{6}{|c|}{ Internet } \\
\hline & & Fijo & $\%$ & Móvil & $\%$ & Fax & $\%$ & Fijo & $\%$ & Móvil & $\%$ & $\begin{array}{l}\text { Otras } \\
\text { redes }\end{array}$ & $\%$ \\
\hline Argelia & 7 & 2 & 29 & 7 & 100 & 2 & 29 & 2 & 29 & 3 & 43 & 2 & 29 \\
\hline Bulgaria & 18 & 4 & 22 & 18 & 100 & 4 & 22 & 9 & 50 & 16 & 89 & 3 & 17 \\
\hline Colombia & 28 & 6 & 21 & 28 & 100 & 6 & 21 & 23 & 82 & 28 & 100 & 5 & 18 \\
\hline Ecuador & 59 & 10 & 17 & 59 & 100 & 7 & 12 & 26 & 44 & 40 & 68 & 6 & 10 \\
\hline Malí & 16 & 3 & 19 & 16 & 100 & 0 & 0 & 2 & 13 & 6 & 38 & 5 & 31 \\
\hline Marruecos & 36 & 5 & 14 & 36 & 100 & 2 & 6 & 7 & 19 & 28 & 78 & 2 & 6 \\
\hline Rumania & 24 & 6 & 25 & 24 & 100 & 0 & 0 & 8 & 33 & 23 & 96 & 6 & 25 \\
\hline Senegal & 28 & 6 & 21 & 28 & 100 & 1 & 4 & 5 & 18 & 15 & 54 & 5 & 18 \\
\hline Total & 216 & 42 & 19 & 216 & 100 & 22 & 10 & 82 & 38 & 159 & 74 & 34 & 16 \\
\hline
\end{tabular}

Fuente: elaboración propia.

En este panorama, las nuevas tecnologías se presentan como una oportunidad y al mismo tiempo como una amenaza, en especial cuando la brecha digital toca sus puertas (prueba de ello fue el análisis de las TIC encontradas en el tejido asociativo inmigrante, puesto que los resultados sugieren que el $100 \%$ de las asociaciones cuentan con líneas móviles como herramienta indispensable de trabajo, estas líneas no siempre son de uso exclusivo de la asociación; de hecho, se detectó que más del 50\% de esas líneas son de uso personal (se "presta” el número, puesto que son líneas particulares que tienen una doble funcionalidad). Este comportamiento hace parte de una de las tantas estrategias para 
afrontar la crisis económica actual, pero también supone una disminución en la calidad del trabajo de las asociaciones.

Algunas de las consecuencias de la utilización de los móviles y otros dispositivos son la no diferenciación de lo profesional y lo personal. De hecho, si una persona usa su línea telefónica fija o móvil para dos fines en el que uno de ellos está contemplado la vida laboral, no podrá establecer límites y desconectar. Prueba de ello, fue que, durante la investigación en la etapa de contacto se llamó a los números de las asociaciones con el resultado de que en el caso de líneas fijas contestaban familiares del responsable de la asociación, quienes nos proporcionaron un número móvil (asimismo, facilitaban información adicional que puede ser errónea o mal interpretada). En el caso de los móviles, muchas de las personas que en la actualidad representan la asociación, contestan sus líneas desde otros trabajos, poniendo en peligro su propia estabilidad y la de asociación, ya que no pueden brindar un buen servicio al usuario bajo estas condiciones (Tovar y Torralba, 2016).

El porcentaje de incorporación de redes sociales de comunicación y acceso a internet es muy limitado, en especial porque el $74 \%$ de las asociaciones se conectan a través del móvil; estos dispositivos, por lo general, no pertenecen a la asociación, sino que al igual que los demás implementos, pertenecen a los miembros de las asociaciones, quienes ponen sus aparatos y redes personales en uso de la asociación, para de esta forma ahorrar dinero.

En cuanto al acceso a internet a través del móvil y del ADSL, se puede concluir que no existe una calidad en la conexión, ya que no se accede a los mismos contenidos y no se cuenta con el mismo ancho de banda, estando condicionada a los megas que contraten, a la calidad de los dispositivos y al uso de una sede propia.

Tabla 2. Redes sociales y el tejido asociativo

\begin{tabular}{|l|c|c|c|c|c|c|}
\hline \multirow{2}{*}{ País } & \multirow{2}{*}{ Frecuencia } & Página web & Blog & Facebook & Twitter & YouTube \\
\cline { 3 - 7 } & & $\mathbf{S i}$ & $\mathbf{S i}$ & $\mathbf{S i}$ & $\mathbf{S i}$ & $\mathbf{S i}$ \\
\hline Argelia & 7 & 1 & 1 & 2 & 0 & 0 \\
\hline Bulgaria & 18 & 6 & 5 & 12 & 2 & 2 \\
\hline Colombia & 28 & 15 & 2 & 11 & 4 & 7 \\
\hline Ecuador & 59 & 22 & 18 & 34 & 11 & 12 \\
\hline Mali & 16 & 1 & 1 & 1 & 0 & 0 \\
\hline Marruecos & 36 & 12 & 5 & 12 & 5 & 4 \\
\hline Rumania & 24 & 9 & 0 & 5 & 0 & 0 \\
\hline Senegal & 28 & 3 & 2 & 2 & 0 & 1 \\
\hline \multirow{2}{*}{ Total } & 216 & 69 & 34 & 79 & 22 & 26 \\
\cline { 3 - 7 } & & $32 \%$ & $23 \%$ & $43 \%$ & $16 \%$ & $13 \%$ \\
\hline
\end{tabular}

Fuente: elaboración propia.

Como se observó en la investigación, el porcentaje de incorporación de las redes sociales como herramienta de ayuda en las asociaciones resulta más bien bajo, cuando un $43 \%$ de las asociaciones usan Facebook, 32\% tiene páginas web, 23\% blogs, 16\% Twitter y un $13 \%$ usan YouTube (Tabla 2).

Estos resultados indican que las asociaciones hacen un uso parcial y muy limitado de las nuevas tecnologías, lo que no les permite desarrollar todo su potencial, en especial porque la situación socioeconómica y política actual del país ha repercutido en el tejido asociativo 
inmigrante en España, al igual que la falta de preparación y formación en tecnologías, al punto de constituirse en uno de los principales retos y obstáculos para la integración de las TIC y su inclusión en la sociedad de la información.

En este sentido, podemos decir que aún falta capacitación para mejorar su aprovechamiento, ya que son redes de contacto que necesitan ser actualizadas casi a diario, en especial por los tiempos de respuesta y la calidad de sus contenidos; además, dependen de la pericia que se tenga en la administración de los contactos, lo que afecta también al aumento de sus seguidores.

En general, Facebook se presenta como la red social más utilizada a nivel del tejido asociativo, en especial porque es una red gratuita de acceso generalizado, tanto en España como en los países de origen de los flujos migratorios, que es de fácil acceso y puede ser utilizada para gran variedad de fines, entre los que sobresale la comunicación, la publicidad y como red de captación de socios. También es una red fundamental para el intercambio de información con los países de origen y con los usuarios que forman parte de sus redes de contacto, acerca de sus actividades interculturales, deportivas, fiestas y religiosas. Además, es una red que está habilitada a la mayor cantidad de dispositivos posibles, destacándose el móvil, la tableta y el ordenador.

Las páginas web y las redes sociales son excelentes instrumentos de divulgación de información; sin embargo, no es lo mismo tener páginas de pago creadas por profesionales que aquellas que son gratuitas, especialmente si no se tienen las habilidades y destrezas para hacer un excelente portal (aunque en internet se tienen las herramientas y los tutoriales de cómo elaborarlas, las asociaciones no tienen esa pericia o formación para intentar crear sus páginas en este tipo de portales).

También encontramos páginas inactivas y obsoletas que aún siguen de alta en la web y que no han sido dadas de baja por el proveedor. Las asociaciones, lejos de advertir un problema, consideran que es una oportunidad, razón por la cual tampoco exigen al proveedor la baja. Esta situación se convierte en un problema para la asociación que, sin saberlo, está maximizando la pérdida de asociados, en tanto que la información es obsoleta, poniendo así en juego su credibilidad y fiabilidad como asociación.

Aunque existen páginas web y blogs gratuitos, la demanda hecha por las asociaciones hacia esta iniciativa es más bien escasa y las asociaciones que han intentado construir su página las han abandonado en el proceso, por esta razón cuando se hace la búsqueda en la web aparecen las páginas con el letrero que indica "en construcción”.

En cuanto al uso y administración de las páginas web y redes sociales, se hizo evidente la falta de capacitación y conocimiento del manejo de esas herramientas, puesto que existe poca claridad en cuanto a las características y diferenciación de cada red, razón por la cual se tiende a utilizar todas de manera uniforme, cayendo en el error de publicar la misma información en todas las páginas y acabando por afectar la eficacia del mensaje que se quería transmitir.

La información publicada en la mayoría de las páginas web y las redes sociales de las asociaciones estaba desactualizada y carecía de material propio (en su defecto se utilizaba material de otras páginas). También se evidencia la saturación de información y enlaces rotos que no conducían a ninguna página. Por lo general, no se hace conexión entre las redes de la misma asociación, y esto es debido a la falta de creación de contenido propio, así como de formación al respecto.

Fue evidente la falta de formación en cuanto a criterios de selección a la hora de sumarse a otras redes de contactos, puesto que a nivel general se encontró que las asociaciones 
tienden a sumarse a un gran número de redes de otras entidades y personas, sin hacer diferenciación entre los objetivos de una red personal y los de una red laboral; de esta forma, buena parte de la información que se recibe carece de calidad para la organización y sus beneficiarios, por este motivo, el número de personas o entidades interesadas por sus propias redes es más bien escasa.

También se evidenció la falta de conocimiento y control de los cronómetros y estadísticas acerca del número de visitas, de registrados, comentarios y de información sobre el número de veces que una publicación de la página es compartida en otras redes sociales, puesto que ellas sirven de bitácora en la pertinencia de las publicaciones.

Asimismo, de estos criterios depende la imagen que los usuarios se hagan de la asociación, lo que también puede incidir en el número de afiliados.

El correo electrónico se sitúa como uno de los instrumentos con mayor número de usuarios, a razón de ser considerado una de las mayores fuentes de trasferencia de información gratuita e instantánea, convirtiéndose en una de las principales herramientas ineludibles para todo aquel que quiera hacer uso de las sedes electrónicas, así, como de las redes sociales y servicios de la web (Tabla 3).

Tabla 3. Correos electrónicos (E-mail)

\begin{tabular}{|l|c|c|c|c|}
\hline \multicolumn{1}{|c|}{ País } & N. $^{\mathbf{0}}$ de Aso. & E-mail de la asociación & E-mails personales & E-mail con dominio \\
\hline Argelia & 7 & 5 & 4 & 0 \\
\hline Bulgaria & 18 & 3 & 6 & 2 \\
\hline Colombia & 28 & 11 & 3 & 7 \\
\hline Ecuador & 59 & 46 & 14 & 6 \\
\hline Mali & 16 & 3 & 6 & 0 \\
\hline Marruecos & 36 & 22 & 8 & 8 \\
\hline Rumania & 24 & 14 & 6 & 1 \\
\hline Senegal & 28 & 9 & 2 & 3 \\
\hline Total & 216 & 113 & 49 & 27 \\
\hline Total \% & 100 & $52 \%$ & $23 \%$ & $12,50 \%$ \\
\hline
\end{tabular}

Fuente: elaboración propia.

Actualmente, la mayoría de los dispositivos, en especial los móviles de tercera y cuarta generación, utilizan el correo electrónico como puerta obligatoria de acceso, debido a las grandes alianzas estratégicas de Google y el sistema operativo Android. Estas alianzas también influyen en los usuarios, ya que determinan la apertura del correo al momento de utilizar la tecnología.

Los resultados de la investigación indican que el 100\% de las asociaciones tienen operativa una cuenta de correo electrónico como herramienta de trabajo diario. De ese porcentaje, el 52\% cuentan con correos que cumplen con la condición de conservar el nombre o siglas de la asociación, mientras que el 22\% son cuentas de tipo personal.

$\mathrm{Al}$ respecto, las asociaciones deberán de controlar sus cuentas de correo y las claves de acceso creadas para dicho fin, puesto que buena parte de estas cuentas fueron creadas por personas que en su momento ejercían algún tipo de cargo o función en la organización, y cualquier modificación en esta situación conlleva la pérdida de contactos, ya sea por inactividad de la cuenta e incluso sustracciones o sabotajes en las bases de datos. 
Las asociaciones deben de concientizarse de la importancia que tiene para sus organizaciones la creación y mantenimiento de las bases de datos, ya que constituyen fuentes de recursos económicos y de información, razón por la cual deberán de administrar custodiar, actualizar, aumentar y mantener sus contactos como parte del capital social y económico de la asociación.

La ventaja es que permite tener mayor control sobre el uso del correo y de los contactos, ya que estas cuentas se crean con el inicio de actividad y se cierran con su cese, sin que ello repercuta en la base de datos, puesto que la información se almacena en el servidor de la empresa (al respecto se encontró que el 12.5\% de las asociaciones cuentan con dominio propio, excluyendo las asociaciones de Argelia y Malí quienes no cuentan con este tipo de cuentas de correo).

El que una asociación tenga o no un dominio propio describe en cierta medida la capacidad económica y adquisitiva de la organización, encontrando que la mayor parte de ese tipo de correos electrónicos pertenecen a asociaciones con reconocimiento en el entorno por su trayectoria de trabajo superior a 10 años y mayores recursos.

En términos generales, se detectaron profundos problemas en la diferenciación de las herramientas y recursos laborales de los personales, ya que la investigación encontró que el mayor número de cuentas en las redes sociales y correos electrónicos están a nombre de una tercera persona y no de la asociación, razón por la cual no fueron incluidos en las bases de datos.

En cuanto a la inclusión de las tecnologías de información y comunicación TIC en los países de origen y destino se concluyó que las mismas estarán condicionadas por el nivel de desarrollo y de implantación tecnológica con la que cuente el país de origen, así como por la situación de conexión en el mundo. Al respecto la investigación se encontró con una gran barrera en cuanto a la calidad y afluencia de la información, puesto que no todos los países publican esta información y la que está disponible es más bien general y escasa, no haciendo énfasis en las particularidades de cada país.

Además, se deberá de tener en cuenta que buena parte de los flujos migratorios estudiados provienen de zonas rurales azotadas por problemas de inclusión e implantación digital. Igualmente, sería necesario evaluar con mayor profundidad la inclusión y participación de las asociaciones en origen y destino a la luz de los contactos de cada una de las redes, puesto que en ellas participan personas y entidades procedentes de países de origen y destino. Al respecto se considera que las redes sociales logran convocar, crear y mantener redes de contactos en los países de origen y destino.

\section{CONCLUSIONES}

A partir del empleo de las tecnologías de comunicación e información se hace visible cómo la brecha digital afecta el tejido asociativo inmigrante y el abismo que existe entre las asociaciones “conectadas” y las asociaciones “no conectadas”. Las nuevas tecnologías son fundamentales para el desarrollo actual del asociacionismo inmigrante, y este último sigue siendo clave para favorecer la integración de los inmigrantes.

Las asociaciones estudiadas muestran en conjunto un considerable número de iniciativas, aunque con diferencias significativas entre los diferentes colectivos, de acuerdo con los recursos de que disponen y el uso e incorporación de las tecnologías de información y comunicación para lograrlo. 
En el caso de las asociaciones del Magreb, el número de inmigrantes marroquíes en España es mayor que el de los argelinos, lo que explica que Marruecos tenga mayor número de asociaciones y número de proyectos dirigidos hacia su país de origen, mientras que las asociaciones argelinas tienen baja capacidad asociativa y de participación en origen.

En cuanto a sus instalaciones, se encontró que el 39\% de las asociaciones argelinas no tiene local y solo una mínima parte cuenta con locales cedidos que, en general, son instalaciones pertenecientes a entes públicos, mientras el número de asociaciones marroquíes sin local es menor. A nivel de personal, se determinó que ambos países utilizan la figura del voluntariado para el desempeño y funcionamiento de las tareas de la asociación. Sin embargo, las asociaciones marroquíes son las que mayor número de personal contratado tienen, aunque los dos colectivos utilizan las páginas web para el reclutamiento de personal voluntario.

La incorporación de redes y medios de difusión social es bastante reducida entre el tejido asociativo argelino en comparación con el tejido asociativo marroquí. De hecho, solo se encontraron dos asociaciones en Facebook, un blog y una página web argelina; mientras que las asociaciones de Marruecos tienen incidencia en todas las redes y medios de difusión, siendo Facebook y las páginas web las más demandadas. Los servicios de telefonía fija y móvil en ambos colectivos son equitativos, así como el uso del internet, siendo el internet móvil el sistema líder por excelencia.

Las asociaciones de América Latina (Colombia y Ecuador) forman parte de colectivos con amplia representación en España. Estos colectivos han logrado, a través del desarrollo de proyectos en sus países de origen, convertirse en ejemplo de buenas prácticas para otros colectivos, consiguiendo crear asociaciones con capacidad para impulsar sus proyectos.

El número de asociaciones ecuatorianas es más numeroso que el de las colombianas, dada su mayor población en España y, en parte, porque su experiencia migratoria es más antigua. Estas asociaciones han centrado sus esfuerzos en desarrollar proyectos sobre la mejora y la dotación de infraestructuras, turismo solidario, y cooperativismo.

Las asociaciones ecuatorianas y colombianas cuentan con sede para el funcionamiento de su asociación, ya sea porque han alquilado un espacio, se los han cedido, o porque comparten la sede con otras instituciones; sin embargo, a diferencia de las asociaciones ecuatorianas, las asociaciones colombianas tienen un mayor porcentaje de sedes propias para su funcionamiento.

Con respecto al personal se encontró que ambos colectivos utilizan la figura del voluntario en sus asociaciones, aunque el mayor número de contratados se encuentra en las asociaciones colombianas. Estos dos colectivos son los que más usan las páginas web para convocar personal voluntario, especialmente las asociaciones de Ecuador.

La participación digital de ambos colectivos es alta, siendo Facebook la red más usada, al igual que las páginas web, salvo que la utilización de los blogs por parte de las asociaciones ecuatorianas es mayor. El uso de telefonía móvil, telefonía de línea fija y el fax en las asociaciones de estos colectivos es similar, así como el uso de internet móvil y fijo.

En África Subsahariana (Senegal y Mali) las diferencias son notables. Senegal tiene una inmigración más antigua y asentada, pero la migración de Mali es más reciente y vulnerable. De este modo, más de la mitad de las asociaciones malienses carecen de local, y tan solo una mínima parte cuenta con locales cedidos en general por ayuntamientos, mientras que las asociaciones senegalesas cuentan con locales alquilados, cedidos, prestados o propios para el desempeño de sus actividades. 
En materia de personal se encontró que ambos colectivos utilizan la figura del voluntario, pero a nivel de contratos los datos muestran que Senegal está muy por encima de Malí. En estos dos colectivos, aunque utilizan las páginas web para convocar voluntarios para su asociación, su representatividad y utilidad son bastante reducidas, teniendo en cuenta el total de asociaciones.

La utilización de redes y medios de difusión social en las asociaciones de Malí es bastante reducida, de hecho, solo se encontró registro de una cuenta en Facebook y una página de blog del total de las asociaciones, mientras que el uso de redes y medios de difusión social por parte del tejido asociativo senegalés es mayor y más activo. El uso de telefonía móvil y de línea fija es similar en los dos colectivos: menor uso de line fija y mayor uso de la telefonía móvil, en cuento al uso de internet a través de ADSL o red móvil se encontró que es reducido, especialmente el de las asociaciones malienses, además la mayoría de las conexiones de ambos colectivos se realizan a través del teléfono móvil.

Los países de Europa del Este (Bulgaria y Rumanía) se caracterizaron principalmente por ser una migración económica que llego a España atraída el mercado de trabajo. Sin embargo, con la crisis, y completada la adhesión de estos países a la Unión Europea, aumentan las salidas de esta población de España, especialmente la población rumana.

Las asociaciones de Bulgaria y Rumanía, han centrado sus acciones en proyectos a favor del mantenimiento de su cultura de origen como prioridad.

A nivel general estas asociaciones cuentan con sedes propias, alquiladas o cedidas; sin embargo, el tejido asociativo búlgaro es el que cuenta con mayor número de sedes propias, incluso se encuentra por encima del resto de colectivos y, al igual que Malí, no comparte espacios para el funcionamiento de sus asociaciones.

Ambos, colectivos cuentan con la figura del voluntariado y también más de la mitad de estas asociaciones cuentan con personal contratado. Solo una parte muy reducida de las asociaciones búlgaras utilizan las páginas web para la promoción del voluntariado.

El uso de redes y medios de difusión social por parte del tejido asociativo búlgaro es mayor que el de las asociaciones rumanas; además, las asociaciones búlgaras tienen cuentas en todas las redes de difusión social, mientras que el tejido asociativo rumano solo tiene cuentas en Facebook y algunas páginas de blog. El uso de la telefonía móvil y del internet en las asociaciones de los dos países es similar: existe mayor número de líneas móviles y menor uso de líneas fijas, así como las conexiones a internet se realizan en mayor medida a través de redes telefónicas móviles que a través de ADSL fija.

Como se podrá observar a partir de las tecnologías de comunicación e información se hace visible como la brecha digital afecta el tejido asociativo y el abismo que existe entre las asociaciones “conectadas” y las asociaciones "no conectadas” a partir de las tres accesiones básicas: acceso, uso y contenido. De esta forma encontramos que la misma logra generar una diferencia entre las asociaciones que cuentan con los dispositivos tecnológicos, de conexión a internet y de redes avanzadas como servidores de las asociaciones que no.

\section{RECOMENDACIONES}

Se recomienda incentivar la investigación en temas relacionados con las tecnologías de información y comunicación, así como en temas relacionados con las redes y medios 
sociales en el tercer sector. Su aplicabilidad en el área social, a través de un seguimiento sobre la evolución de las asociaciones existentes, así como del tipo de tecnologías que han incorporado en sus organizaciones y en los proyectos que desarrollan, tanto en sus países de origen como los países de destino.

Es necesario seguir investigando en el tema e incluir variables como edad, genero, nivel de escolaridad, nivel de ingresos, ya que de esta forma se podría desarrollar programas de capacitación especializados.

Se insta a todas las asociaciones a abrir sus propios canales de YouTube, así como sus propias cuentas en cada red social a nombre de la institución. De otra forma los beneficios de las publicaciones se pierden y, en caso de que el personal no continúe en la organización, esta información y sus contactos pueden desaparecer; además, en la red de YouTube el número de visualizaciones puede generar ingresos para las asociaciones, lo que constituye un valioso aporte económico.

Pese a todas las dificultades señaladas las asociaciones inmigrantes deben hacer un mayor esfuerzo en el empleo de las TIC como una estrategia de inclusión social de la población inmigrante.

\section{REFERENCIAS}

Comisión de Ciudades Digitales (2006). Manual de ciudades digitales.

Comisión Europea (2010). Comunicación de la Comisión Europa 2020. Una estrategia para un crecimiento inteligente, sostenible e integrador.

Fundación acción contra el hambre (2002). Conceptos: Pobreza, Exclusión Social, Vulnerabilidad e Inclusión Social Madrid. Recuperado de: http://www.fundacionluisvives.org/temas/inclusion_social/conceptos_pobreza exclusion_social_vulnerabilidad_e_inclusion_social (20/05/2020).

Maitland, D. (1984). El eslabón perdido. I informé de la comisión independiente para el desarrollo mundial de las telecomunicaciones,.

Ministerio de Asuntos Exteriores y Cooperación (2013). Plan Director de la cooperación Española (2013-2016).

Mochón, F. (2006). Globalización retos de cara al futuro. Cuadernos de cc.ee. y ee., (50), 51-56.

Morales, J., y Rodríguez, M. (2002). VI Sexto Congreso de sociología, Mundos sociales saberes y prácticas, Universidad de Lisboa.

Objetivo de desarrollo sostenible (2016-2030). 17 objetivos para trasformar el mundo. Recuperado de http://www.un.org/sustainabledevelopment/es/objetivos-dedesarrollo-sostenible/ (20/05/2020).

Rodríguez, P. (2012). La tercera brecha digital: ¿Es conocimiento todo lo que reluce en el internet? Recuperado de: http://blogthinkbig.com/es-conocimiento-todo-lo-quereluce-en-internet/ (20/05/2020).

Serrano, S., y Martínez, E. (2003). La brecha digital: Mitos y realidades. Universidad Autónoma de Baja California, México. 
Tovar, J. C. (2013). Las nuevas tecnologías de información y comunicación (TIC) y el tejido asociativo de emigrantes en España. En la migración circular. Comares, Valencia.

Tovar, J. C., y Torralba, J. (2016). Nuevas tecnologías de información y comunicación TICS en el tejido asociativo inmigrante de España. Revista iberoamericana Unizar, 1-17. Recuperado de: http://ried.unizar.es/index.php/revista/thesis/view/29 (20/05/2020).

Unión Internacional de Telecomunicaciones (2005). CMSI Documentos finales Cumbre mundial sobre la sociedad de la información Ginebra 2003- Túnez 2005. UIT, Ginebra.

Van Dijk, J. (2001). De netwerkmaatschappij: sociale aspecten van nieuwe media. Kluwer, Holanda. 


\section{CONTRIBUCIÓN SEGÚN AUTORES}

\begin{tabular}{|c|l|c|c|}
\hline \multicolumn{1}{|c|}{ ITEM } & Jenny Carolina Tovar & Javier Ernesto Torralba \\
\hline 1 & Conceptualización & $90 \%$ & $10 \%$ \\
\hline 2 & Tratamiento de los datos & $90 \%$ & $10 \%$ \\
\hline 3 & Análisis formal & $90 \%$ & $10 \%$ \\
\hline 4 & Acceso financiación & $90 \%$ & $10 \%$ \\
\hline 5 & Investigación & $90 \%$ & $10 \%$ \\
\hline 6 & Metodología & $90 \%$ & $10 \%$ \\
\hline 7 & Gestión del proyecto & $90 \%$ & $10 \%$ \\
\hline 8 & Recursos & $90 \%$ & $10 \%$ \\
\hline 9 & Software & $90 \%$ & $10 \%$ \\
\hline 10 & Supervisión & $90 \%$ & $10 \%$ \\
\hline 11 & Validación & $90 \%$ & $10 \%$ \\
\hline 12 & Visualización & $90 \%$ & $10 \%$ \\
\hline 13 & Redacción (borrador) & $90 \%$ & $30 \%$ \\
\hline 14 & Redacción final (revisión y edición) & $70 \%$ & $10 \%$ \\
\hline
\end{tabular}

Para más información, visitar CRediT: https:/casrai.org/credit/ 